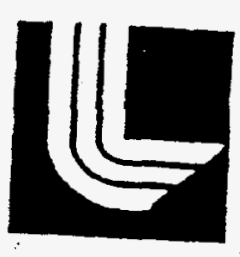

\title{
OPTIONS FOR THE DISPOSITION OF CURRENT INVENTORY OF ROCKY FLATS PLANT RESIDUES
}

\author{
Lychin Chang \\ Lawrence Livermore National Laboratory
}

May 16, 1994

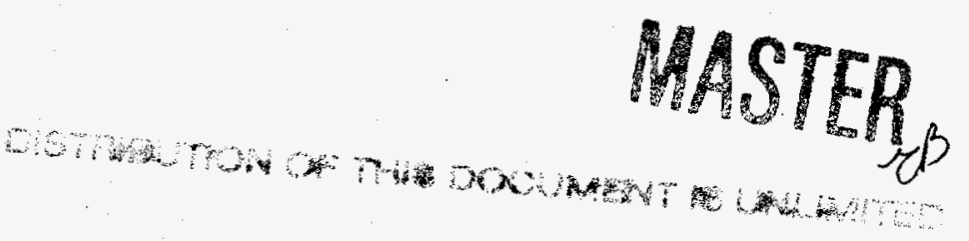




\section{DISCLAIMER}

This report was prepared as an account of work sponsored by an agency of the United States Government. Neither the United States Government nor any agency thereof, nor any of their employees, make any warranty, express or implied, or assumes any legal liability or responsibility for the accuracy, completeness, or usefulness of any information, apparatus, product, or process disclosed, or represents that its use would not infringe privately owned rights. Reference herein to any specific commercial product, process, or service by trade name, trademark, manufacturer, or otherwise does not necessarily constitute or imply its endorsement, recommendation, or favoring by the United States Government or any agency thereof. The views and opinions of authors expressed herein do not necessarily state or reflect those of the United States Government or any agency thereof. 


\section{DISCLAIMER}

Portions of this document may be illegible in electronic image products. Images are produced from the best available original document. 


\section{TABLE OF CONTENTS}

SECTION

PAGE

1.0 STATEMENT OF WORK

2.0 METHODOLOGY

$\begin{array}{ll}3.0 \text { DATA } & 3\end{array}$

4.0 RESULTS

\section{LIST OF TABLES}

Table 1: RFP Residue Feed Stream Summary I-1

Table 2: Estimated Waste Generation $\quad$ I-4

Table 3: Operating Requirements -- Process Wastes vs. Direct Discard I-4

Table 4: Operating Requirements --Waste Categories Processed Individually

\section{LIST OF FIGURES}

Figure 1: Number of Waste Drums vs. Processing Category I-6

Figure 2: Number of LLW Drums vs. Processing Category I-7

Figure 3: Number of TRU Drums vs. Processing Category I-8

Figure 4: Number of Drums - Process vs. Discard Directly I-9

Figure 5: Number of Drums - Process Together vs. Process Individually I-10

Figure 6: Staffing Requirements I-11

Figure 7: Number of Gloveboxes vs. Processing Category I-12

Figure 8: Number of Gloveboxes vs. Strategy I-13

Figure 9: Costs - Process Wastes vs. Discard Directly I-14

Figure 10: Facility Size - Process Wastes vs. Discard Directly I-15

Figure 11: Modified Flowsheets $\quad$ I-16 


\subsection{STATEMENT OF WORK}

With the end of the Cold War, much concern has been directed towards the accumulation of special nuclear material resulting from the dismantlement of a large number of nuclear weapons. This concern has opened up a debate over the final disposition of the large inventory of weapons-capable plutonium. Technologies for the conversion of plutonium into acceptable forms will need to be assessed and evaluated. Candidate strategies for interim and final disposition include a variety of immobilization techniques (vitrification in glass, ceramic, or metal), conversion to reactor fuel, or direct discard as waste. The selected disposition strategy will be chosen based upon a range of decision metrics such as expected conversion costs, equipment requirements, and waste generation.

To this end, a systems analysis approach is necessary for the evaluation and comparison of the different disposition strategies. Current data on inventory of plutonium, such as that at the Rocky Flats Plant (RFP), may be useful for the evaluation and selection of candidate disposition technologies.

A preliminary analysis of the residues of scrap at Rocky Flats was performed to establish a foundation for comparison of candidate strategies. About 3 metric tons of plutonium and 270 metric tons of other wastes remain in the inventory at Rocky Flats. Estimates on the equipment, facility, manpower, and cost requirements to process this inventory over a proposed 10-year cleanup campaign will provide a benchmark for comparison and assessment of proposed disposition technologies.

\subsection{METHODOLOGY}

An integrated computer simulation model was used to obtain estimates of equipment requirements, costs, and wastes generation. The model is based on the process simulation program ProMos (Process Modeling System), the Complex 21 (version 2.2.0) baseline database of process operations, and QuickSim, a spreadsheet model developed for estimation of facility sizing based on plutonium throughput.

A 10-year cleanup campaign is projected for current inventory of Rocky Flats plutonium residues to be processed through a facility. Cost estimates are based upon the assumption of a 13 year construction time for a new facility.

\subsection{DATA}

The summary of the Rocky Flats inventory of plutonium-contaminated residues was taken from the RF IDC list. Wastes were sorted and grouped based upon recommended processing streams, and grouped into categories of:

1) Burn/Cepod/Nitrate,

2) Crush/Cepod/Nitrate

3) Cepod/Nitrate

4) Crush/Burn/Cepod/Nitrate 
5) Crush/Leach/Nitrate

6) Burn/Leach/Nitrate

7) Leach/Drum

8) Leach/Nitrate

9) ER/Chloride

10) MSE/Chloride

11) Crush/Burn

12) Burn/Grout

13) Crush/Grout

14) Grout/Drum

In general, the categories may be further grouped as

1) dissolver recoverable materials (nitrate recoverable materials),

2) salts (chloride recoverable materials),

3) burnable wastes, and

4) materials for direct discard (grouted and drummed).

The categorization of wastes and their accompanying IDC codes are tabulated in Table 1.

For a first estimate, the database for the Complex 21 baseline version 2.2 .0 was adopted. Material is assumed to be processed through the Complex 21 defined processes for nitrate and chloride residue recoveries, and solid and liquid waste treatment. The ProMoS version 2.2.0 database was used for mass balance data, processing times, batch sizes, manpower requirements, and material flow. For the analysis of the Rocky Flats inventory, the materials from the IDC list were categorized and introduced into the appropriate aqueous recovery or waste processing feed operation. With some exception, material was processed along the streams according to the baseline flow diagram. The injection points are indicated in Table 1; the accompanying flowsheets detail the routing of the feed streams and refer to the processing codes summarized in Table 1.

A comparison was made between direct discard of all material versus processing and recovery of plutonium. In Case 1, all wastes are introduced into the facility simultaneously and processed concurrently. Case 1 makes the assumption of uniform plutonium loading in the feed streams, and may be sken as an optimistic estimate of waste generation.

Case 2 represents a more conservative approach. In case 2 , the residues that make up the individual categories (dissolver recoverable materials, salts, burnable wastes, and wastes for direct discard) are grouped, and each category of residue is introduced separately into the facility and processed individually. These values may give a more realistic estimate of facility operations as higher demands are made on facility due to non-uniform and non-optimal loading. Waste generation is expected to be higher for case 2 due to the non-uniform waste aggregation.

Case 3 makes the assumption of no processing of material, and the plutoniumcontaminated residues are directly discarded. Combustibles are burned, as appropriate, and wastes are grouted and drummed directly. 


\subsection{RESULTS}

Comparison of the three cases may be viewed in Tables 2,3 and 4 and the accompanying graphs and charts. Table 2 compares the estimated waste generation among the three cases studies. Table 3 details the facility and operating parameters. Table 4 shows the individual contributions from the recommended processing categories referenced in Table 1.

In general, the following observations are significant:

1) Waste generation:

Direct discard of the IDC list of Rocky Flats inventory would generate nearly a 7 -fold increase in the number transuranic (TRU) waste drums over processing of residues due to plutonium discard limits. Low-level wastes (LLW) under the direct discard option, however, show a significant decrease over the process option since the aqueous lines will not be required.

Under the processing option, dissolver recoverable materials are the largest contributor to the total TRU waste drums. Salts are the largest contributor to LLW waste. Clearly, additional processing/discard alternatives need to be explored.

Processing of all wastes categories together should give a lower bound on waste generation estimates due to the uniform loading of plutonium over the process centers.

2) Costs

Despite the lower facility, equipment and total capital costs under direct discard, total discounted cost for direct discard is estimated to be $33 \%$ higher than if residues are processed. The higher overall cost can be attributed to the higher TRU waste generated under the direct discard option. While capital costs are fixed, the costs for wastes are factored in on an annual basis, thereby increasing the overall discounted cost total.

Discounted costs for the 3 cases may be seen in Table 3 and Figure 9. The discounted cost increases from case 1 to case 2 due to the higher annual waste generation when wastes are the individual categories are processed independently.

\section{3) Facility Size}

As expected, projected facility size is smaller for the direct discard option over the aqueous processing option. Higher capital expenditures are expected if residue processing is chosen over direct discard. 
TABLE 1: RFP Residue Feed Stream Summary

\begin{tabular}{|c|c|c|c|c|c|c|c|c|}
\hline & & & & & & & t & i \\
\hline \multicolumn{9}{|l|}{ Feed Candidate IDCs: } \\
\hline & & & & & & & & \\
\hline & & & & & & & & \\
\hline Processing & & Recommenced Processing & Injection poine & IDCCode & ROCKYFLATSRESTOUE & PU,KG & \multicolumn{2}{|c|}{ TOT MASS, KGITOT MUSS } \\
\hline Categony & & & processing flow & & & & & w/o Pu, KG \\
\hline CSSOLVER RECOVERABLE & 1 & BUPNCEPODNITRATE & A-2, A-1, A-4, etc. & 422 & Soot & 11.7 & 666.1 & 654.4 \\
\hline MATERIALS & & & & 336 & Combustibles, Wet & 33.4 & 69311 & 6897.6 \\
\hline & & & & 330 & Combustibles, Dry & 22.9 & 4589 & 4566.1 \\
\hline & & & & 338 & Filter Media & 93.8 & 2133 & 2038.2 \\
\hline & & & & 331 & Filler, non inc. & 32.11 & 3202 & 3169.9 \\
\hline & & & & 310 & Graph ScartiFines & 76.5 & 944 & 867.5 \\
\hline & & & & 480 & Light Matal & 14.9 & 4034.4 & 4019.5 \\
\hline & & & & & & 285.3 & 22499.5 & 22214.2 \\
\hline & & & & & & & & \\
\hline & 2 & CAUSHCEPDDNITATE & $R-2, R-4$, etc. & 370 & Leco Crucibles & 137 & 8056 & 7919 \\
\hline & & & & & & 137 & 8056 & 7918 \\
\hline & & & & & & & & \\
\hline & & & & & & & & \\
\hline & 3 & CEPOONITRATE & $R-2, R-4$, etc & 159 & Screenings trom Oxide & 137.8 & 320.8 & 183 \\
\hline & & & & 378 & Frebrick Putt/Fines & 13.7 & 291.4 & 277.7 \\
\hline & & & & 420 & Ash, Pull inc. & 695.5 & 9957.2 & 9261.7 \\
\hline & & & & 421 & Ash Hees & 270.31 & 8904.9 & 8634.6 \\
\hline & & & & 290 & Filter Siudge & 14.6 & 379 & 364.4 \\
\hline & & & & 299 & Misc. Sludge & 6.5 & 87 & 80.5 \\
\hline & & & & 340 & Sludge (Size Reduct) & 4.1 & 120 & 115.9 \\
\hline & & & & & & 1142.5 & 20060.3 & 18917.8 \\
\hline & & & & & & & & \\
\hline & 4 & CRUSFVBUPNVCEPOONITRATE & $R-2, R-1, R-4$, etc & 312 & Graphite course & 98.2 & 2129 & 2030.8 \\
\hline & & & & 301 & Class Graph Shapes & 9.3 & 6975 & 6965.7 \\
\hline & & & & 300 & Graphite Molds & $11.8 !$ & 9420 & 9406.2 \\
\hline & & & & & & 119.31 & 18524 & 18404.7 \\
\hline & & & & & & & & \\
\hline & 5 & CAUSHREACHNITRATE & R-2, R-3, R-7, otc. & 377 & Firebrick, Course & 14.7 & 1586 & 1571.3 \\
\hline & & & & 377 & Firebrick, Course & 4.6 & 1717 & 1712.4 \\
\hline & & & & & & 19.3 & 3303 & 3263.7 \\
\hline & & & & & & & & \\
\hline & 6 & BUPUNEACHNITRATE & $R-2, A-1, R-3, R-7$, eic. & 337 & Plastios & 5.1 & 1262 & 1256.9 \\
\hline & & & & & & 5.11 & 1262 & 1256.9 \\
\hline & & & & & & & & \\
\hline & 7 & LEACYYORUM & R-3, R-7, W-21, etc. & 341 & Leaded Drybox Gloves & 3.5 & 8499 & 8495.5 \\
\hline & & & & 339 & Lead Glov Non-Acld & 1.6 & 72611 & 7259.4 \\
\hline & & & & 341 & Leaded Drybox Gloves & 1.3 & 398 & 396.7 \\
\hline & & & & 339 & Lead Glov Non-Acid & 0.2 & 104 & 103.8 \\
\hline & & & & & & 6.6 & 16262 & 16255.4 \\
\hline & & & & & & & & \\
\hline & 8 & LEACHNITRATE & R-3, R-7, etc. & 320 & Heavy NON SS Meta & 17.8 & 2098 & 2080.2 \\
\hline & & & & 371 & Firebrick & 8 & $1133 !$ & 1125 \\
\hline & & & & 440 & Glass & 6.9 & $897.4 !$ & 890.5 \\
\hline & & & & & & 32.7 & $4128.4 !$ & 4095.7 \\
\hline & & & & & & & & \\
\hline SALTS & 9 & ERCHLOPIDE & R-16. etc. & 392 & Unpus Send, Slag \& Cruc & $57.5 !$ & 1562 & 1504.5 \\
\hline & & & & 391 & Unpul Sand a Cruc & 28.41 & 744 & 715.6 \\
\hline & & & & 418 & Mon SallPack LANL & 16.61 & $80.8 !$ & 64.2 \\
\hline & & & & 393 & Send Slag. Cruc Hoel & 13.91 & 2439.9 & 2426 \\
\hline & & & & 411 & I & $498.5 !$ & 7159.31 & 6660.8 \\
\hline & & & & 413 & 国 & 85.31 & 618.9 & 533.6 \\
\hline & & & & 414 & DOR & 46.3 & 936.2 & 889.9 \\
\hline & & & & 398 & Pul Sand, Slag \& Cruc & 27.31 & 534 & 506.7 \\
\hline & & & & 365 & Salt - Bad DOA Run & 25.7 & 32 & 6.3 \\
\hline & & i & & 473 & ER Salt for LANL & 15.11 & 203 & 187.9 \\
\hline & & & & 394 & Sand from $\mathrm{BBO}$ & $8.1 !$ & $66.9 \mid$ & 58.8 \\
\hline & & 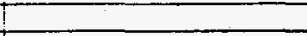 & 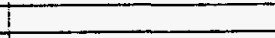 & 415 & Pu Chloride Mixed Salt & 7.11 & 122.6 & 115.5 \\
\hline & & & & 414 & DOA & 6.91 & 3570.41 & 3563.5 \\
\hline & & & & 654 & ER Sall trom PUNP & 6.7 & $54 !$ & 47.3 \\
\hline & & & & 368 & $\mathrm{MgO}$ & 44.8 & 1708 & 1663.2 \\
\hline & & i & & & & 888.2 & 19832 & 18943.8 \\
\hline & & & & & & & & \\
\hline & 10 & MSECHLORIOE & R-15, etc. & 429 & Scrub Alloy Salt & $9.3 !$ & 434.4 & 425.1 \\
\hline & & i & & 409 & NSE & $241.3 !$ & 1398.2 & 1156.9 \\
\hline & & in & 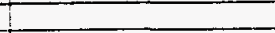 & 427 & IMSE spent Dic Salt & $63.3 i$ & 277.8 & 214.5 \\
\hline & & 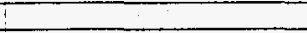 & 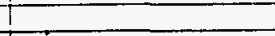 & 405 & IMEE & 32.1 & 1451 & 1418.9 \\
\hline & 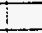 & & & 407 & MSE & 16.51 & $466 !$ & 449.5 \\
\hline & & & 1 & 410 & MSE & 6.6 & 29.3 & 22.7 \\
\hline & $\vdots$ & 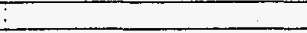 & $i$ & 429 & Scrub Alloy Salt & 5.21 & 1300.6 & 1295.4 \\
\hline & & 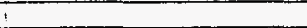 & $i$ & 406 & Mott Salt-Ukn \% Puly & 5.11 & 24.3 & 19.2 \\
\hline & & $\vdots$ & 1 & 408 & MSE & 4.8 & 210 & 205.2 \\
\hline$\cdot$ & & & 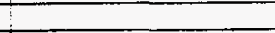 & 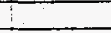 & 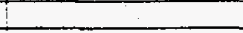 & 384.2 & 5591.6 & 5207.4 \\
\hline & & & & & $!$ & & 1 & 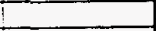 \\
\hline BURNGROUT & 111 & CRUSHBUANGROUT & $W-21, W-22, W-25, W-26$. & 301 & Class Graph Shapes & 0.3 & 2621 & 2620.7 \\
\hline & & & etc. & 312 & Graohile course & $0.3 !$ & $378 !$ & 377.7 \\
\hline & & & 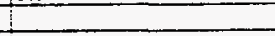 & & & $0.6 !$ & 29991 & 2998.4 \\
\hline & $\bar{i}$ & & 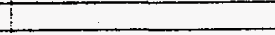 & $i$ & & & 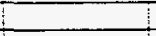 & -2 \\
\hline & 12 & BUAFNGROUT & iw-21, $W-23$, etc. & 485 & iscrap D38 shapes-class & 0.11 & $975 !$ & 974.9 \\
\hline
\end{tabular}


TABLE 1: RFP Residue Feed Stream Summary

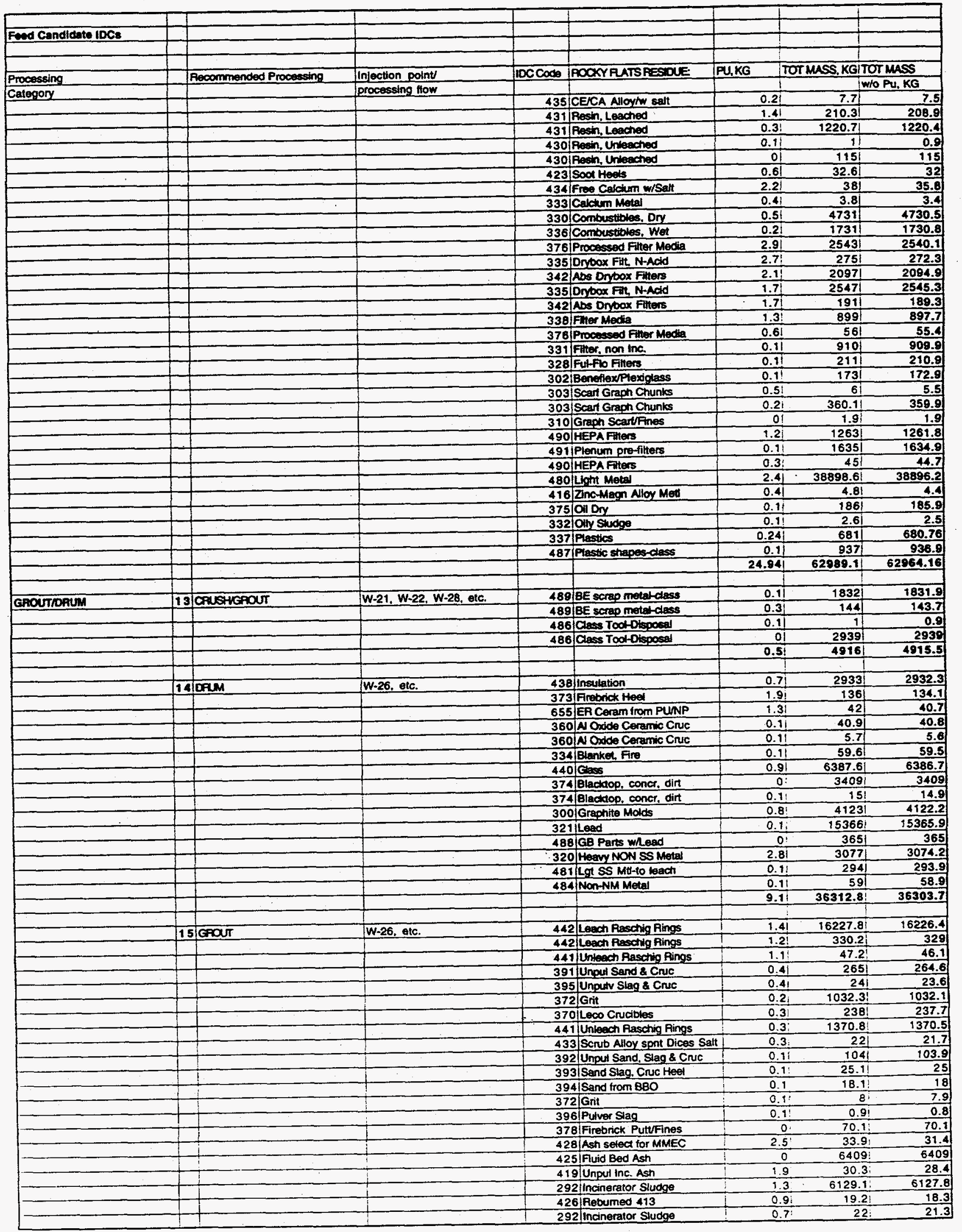


TABLE 1: RFP Residue Feed Stream Summary

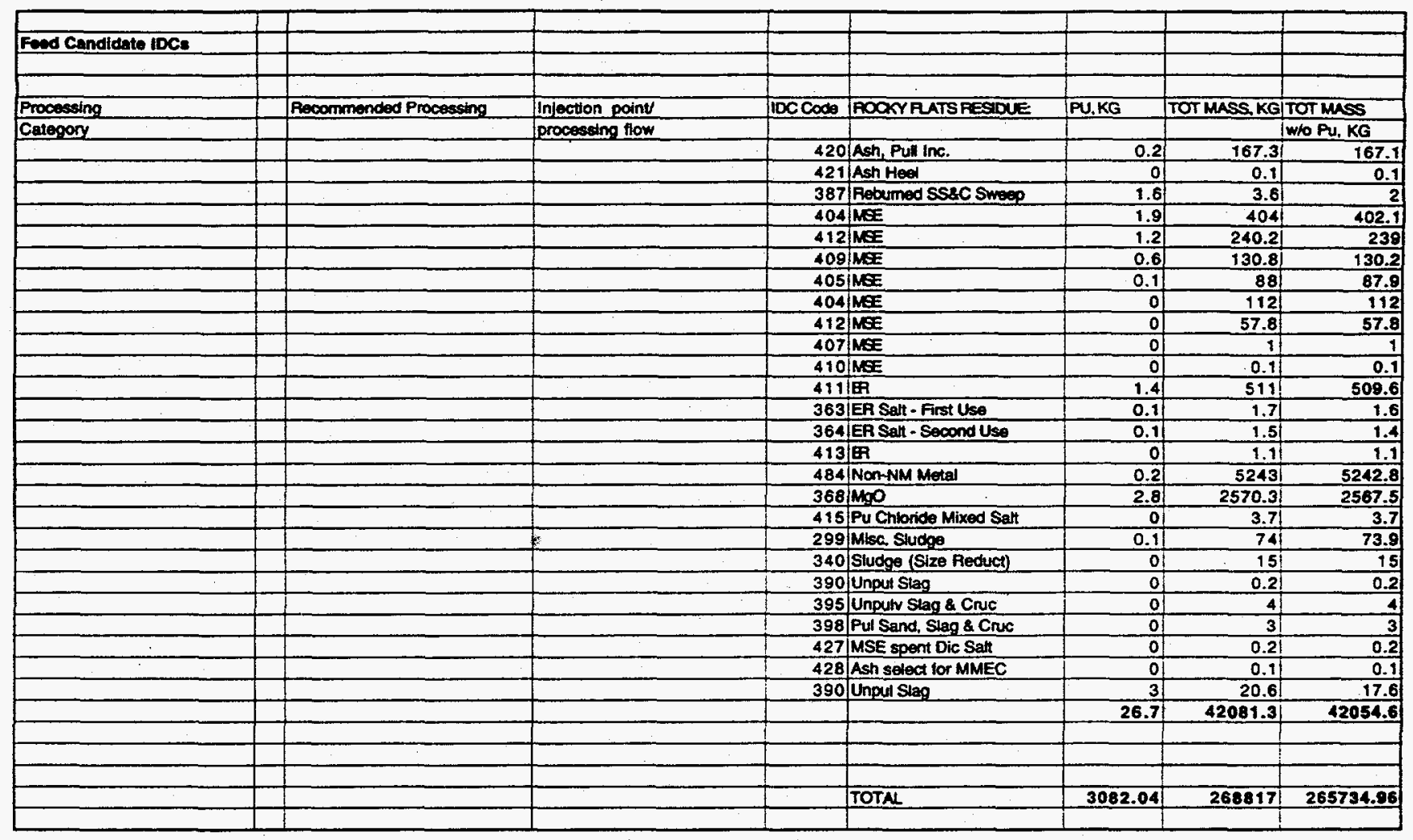


TABLE 2: Estimated Waste Generation

\section{CASE 1: ALL WASTES PFOCESSED SIMUL- TANEOUSLY}

TRU GROUTED WASTE (MT pER year) TRU SOLID WASTE (MT per year) TOTAL TRU DRUMS (Drums/year)

LWW GROUTED WASTE (MT per year)

LIW SOLID WASTE (MT per year)

TOTAL LLW DRUMS (Drums/year)

LQUUID EFFLUENTS (MT/year)

GASEOUS EFFLUENTS (MT/year)

26
90
861
387
17
805

54
15

26

90

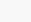

17

805

15
CASE 2: SUMOF WASTES PROCESSED INDNIDUALY
39
171
1252
404
17
854
6
58
16

CASE 3: DISCARD

AL WASTES DIRECTLY WOPROCESSING
0
2020
8392
0
16
56
0
1

TABLE 3: Operating Requirements -- Process Wastes vs. Direct Discard

\author{
CASE 1: ALL WASTES \\ PROCESSED SAMUL- \\ TANEOUSLY
}

122800

42

24

- PROCESSING

- WASTE

- ANALYTICAL LABORATORY

COST

- FACILITY (SM)

- EQUIPMENT (\$M)

- TOTAL CIPITAL (\$M)

OPERATING PARAMETERS

\section{STAFF}

- OPERATING

- SUPPORT

- total

ANNUAL COSTS

- WASTES (\$M)

- OTHER OPERATING COSTS (\$M)

DISCOUNTED COST TOTAL, (\$M)

493

\author{
CASE 2: SUM OF \\ WASTESPAOCESSED \\ INDNIDUALYY
}

122800

42
24
44

234

148

382
CASE 3: DISCARD

ALL. WASTES DIRECTLY

WOPROCESSING

80100

5

46

44

179

108

287

31

183

214

662

773

99

24

1600 
TABLE 4: Operating requirements if wastes categories are processed individually

\begin{tabular}{|c|c|c|c|c|c|}
\hline PERIODIC WASTES & $\begin{array}{l}\text { DISSOLVER } \\
\text { RECOVERABLE } \\
\text { MATERIALS }\end{array}$ & $\begin{array}{l}\text { MATERIALS } \\
\text { PROCESSED } \\
\text { DIRECTLY TO } \\
\text { BURNGROUT }\end{array}$ & $\begin{array}{l}\text { MATERIALS } \\
\text { PROCESSED } \\
\text { DIRECTLY TO } \\
\text { GROUTIDRUM }\end{array}$ & SALTS & $\begin{array}{l}\text { SUMOF } \\
\text { WASTES } \\
\text { PROCESSED } \\
\text { INDIVIDUALLY }\end{array}$ \\
\hline
\end{tabular}

WASTE

TRU GROUTED WASTE (MT per year)

TRU SOLID WASTE (MT per year)

TOTAL TRU DRUMS (Drums/year)

(w/o periodic waste)

LLW GROUTED WASTE (MT per year)

LLW SOLID WASTE (MT per year)

TOTAL LLW DRUMS (Drums/year)

85.1

85.1
847

0

22

39

115.1

0

16.9

61.6

234.8

LIQUID EFFLUENTS (MT/year)

GASEOUS EFFLUENTS (MT/Year)

0.7

18.3

17.6
71
0
0
0
0
0

201

OPERATING PARAMETERS

NUMBER OF GLOVEBOXES

PROCESSING

- WASTE

ANALYTICAL LABORATORY

STAFF

OPERATNG

SUPPORT

-TOTAL

$\begin{array}{cc}4 & 47 \\ 24 & 280\end{array}$

47
280

327

45
271

317

110

662
773 


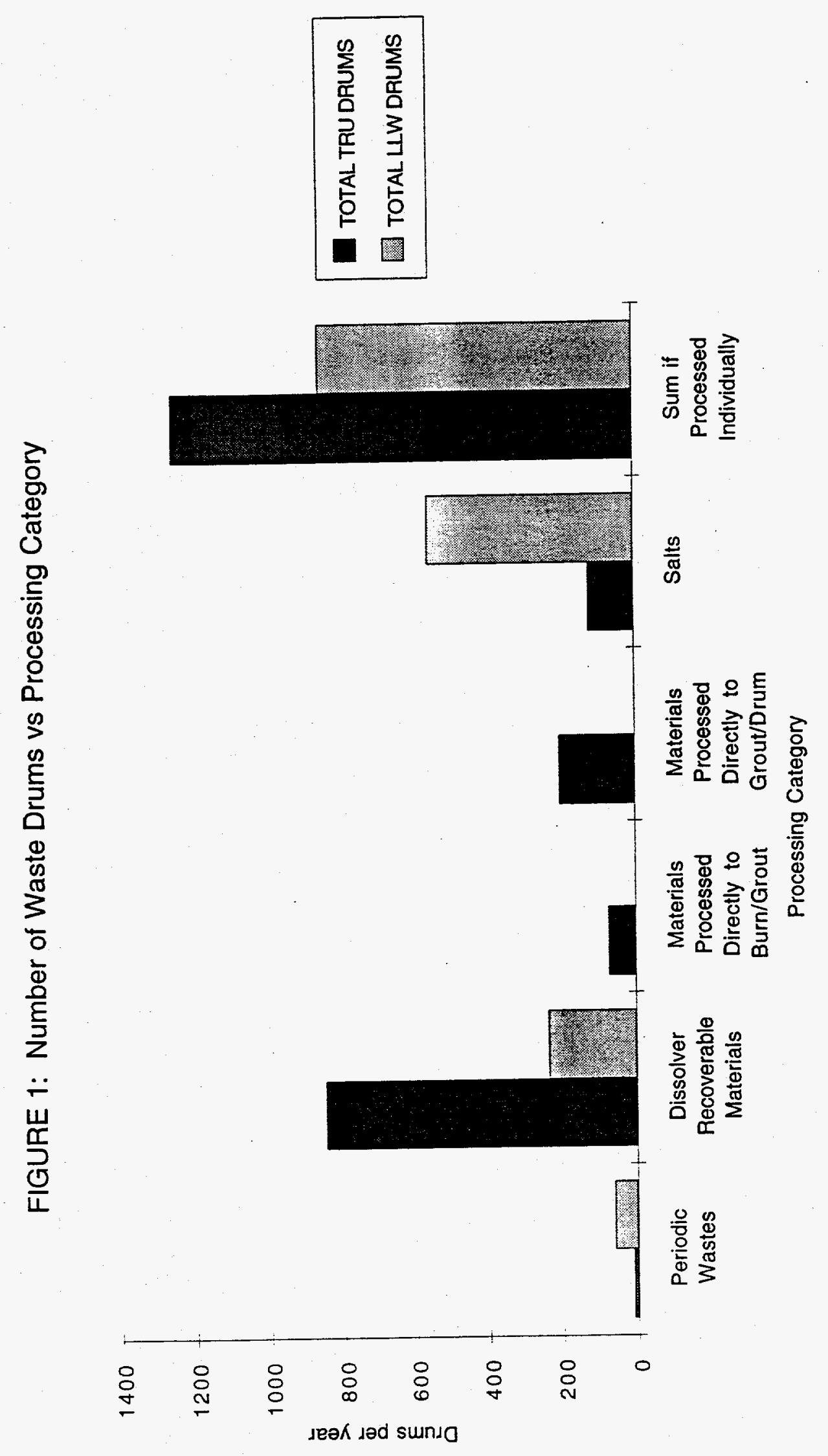


FIGURE 2: Number of LLW Drums vs Processing Category

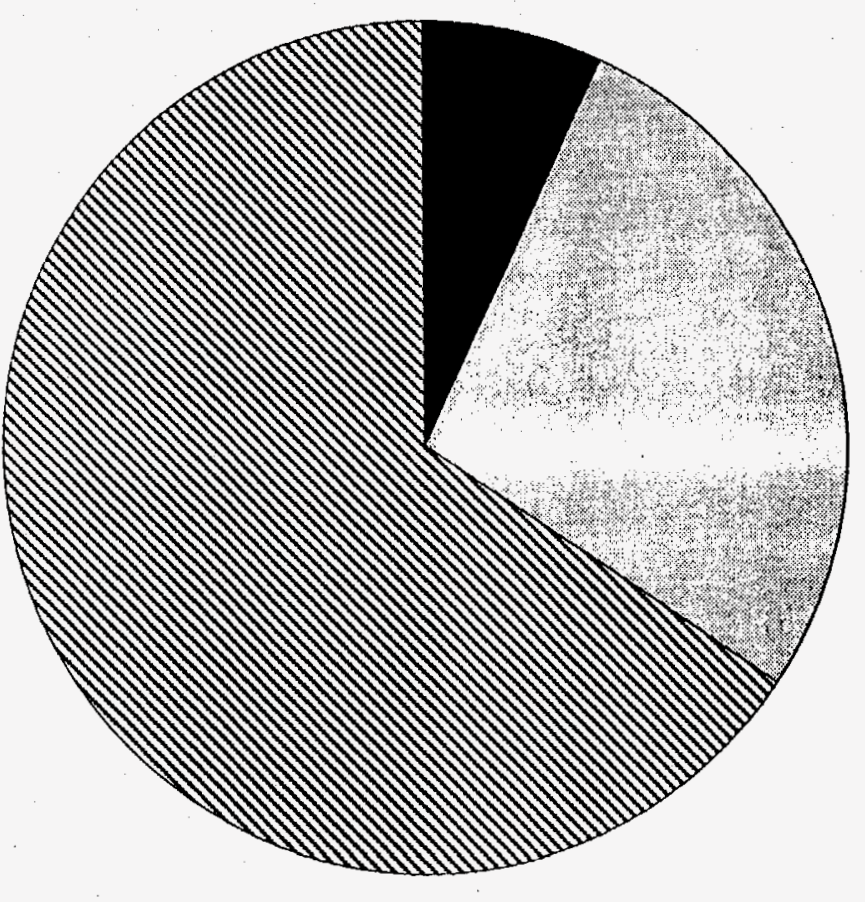

Periodic Wastes

$\square$ Dissolver Recoverable Materials

Materials Processed Directly to Burn/Grout

Materiais Processed Directly to Grout/Drum $\mathbb{N}$ Salts 
FIGURE 3: Number of TRU Drums vs Processing Category

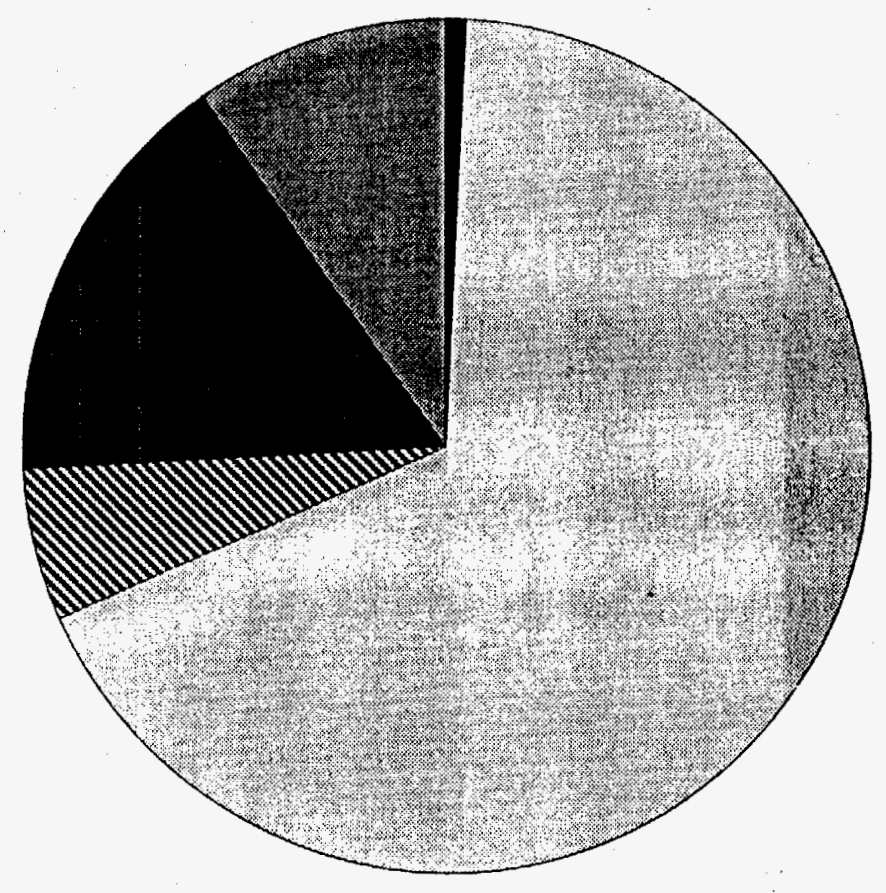

Periodic Wastes

Dissolver Recoverable Materials

Materials Processed Directly to Burn/Grout

Materials Processed Directly to Grout/Drum

Salts 
FIGURE 4: No. of Drums -- Process or Discard Directly

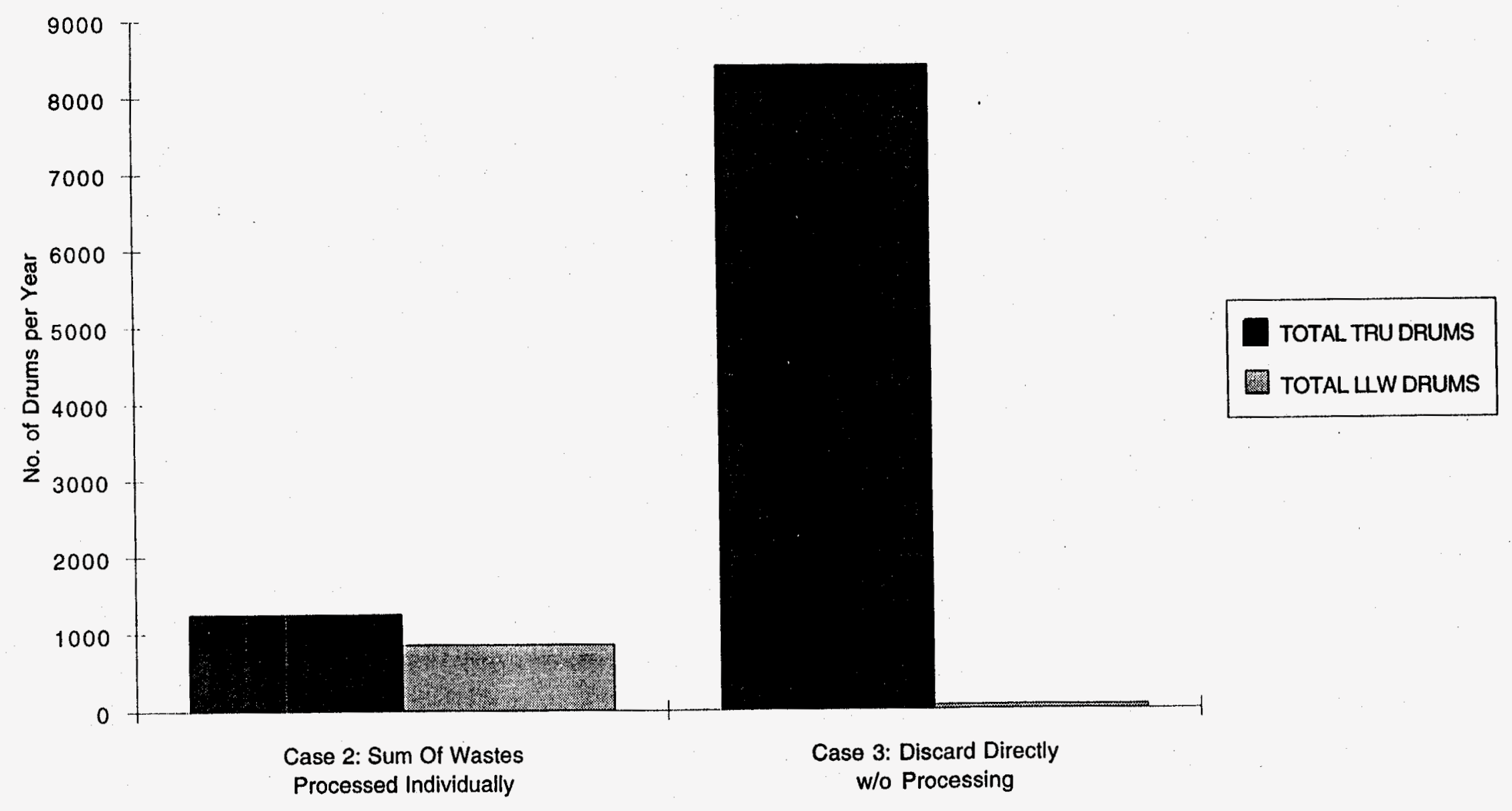


FIGURE 5: No. of Drums - Process Together or Process Individually

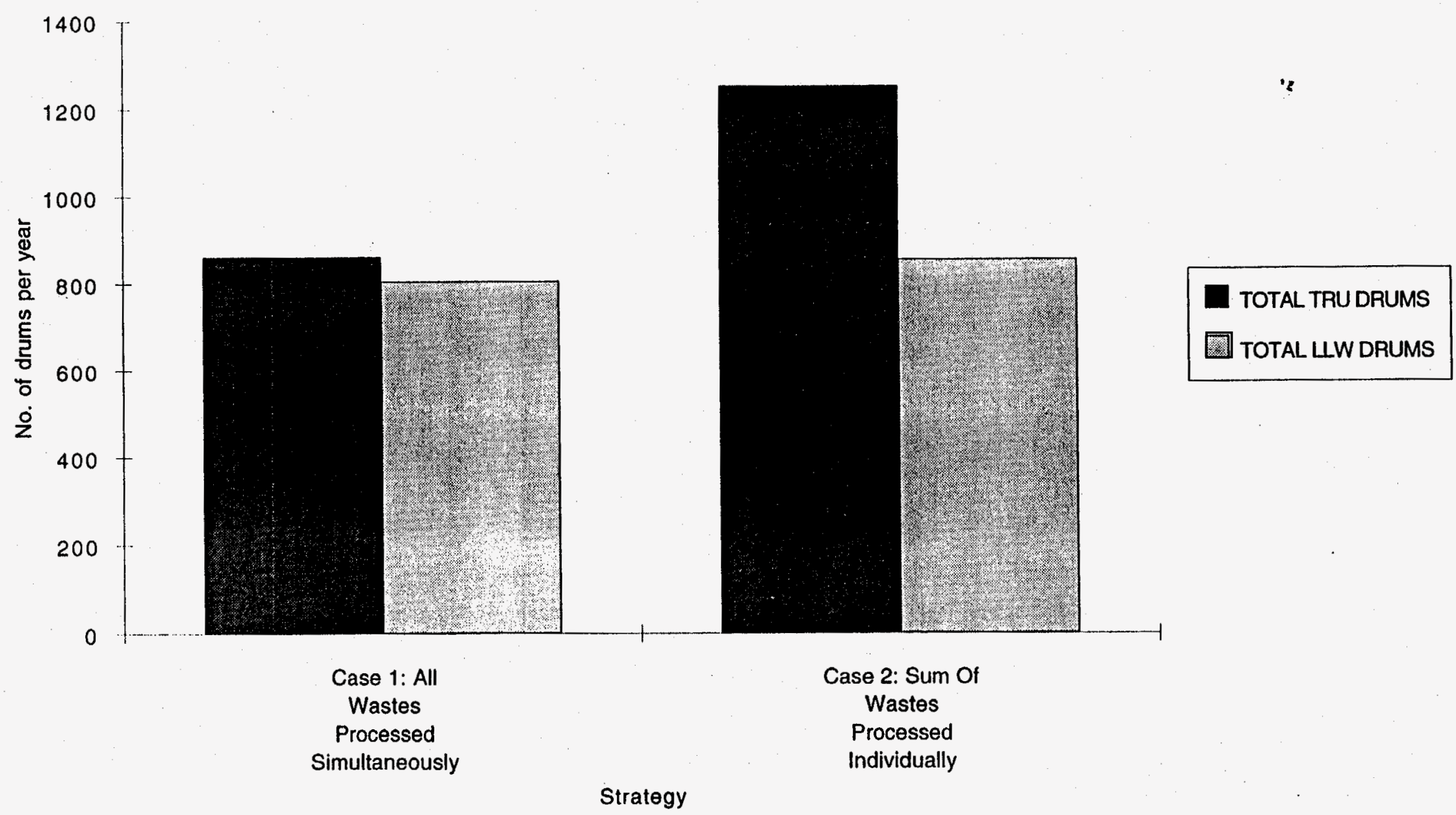





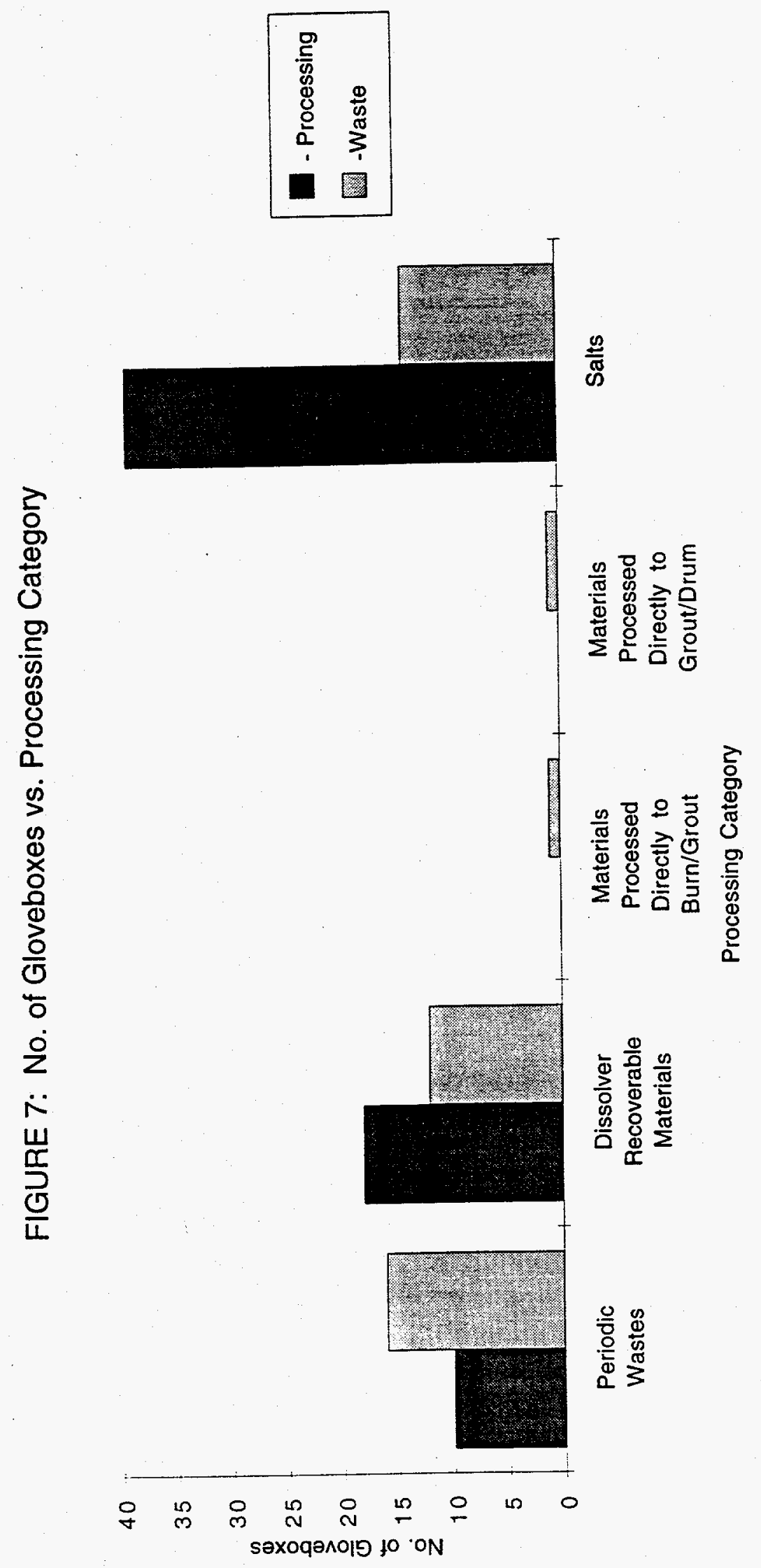




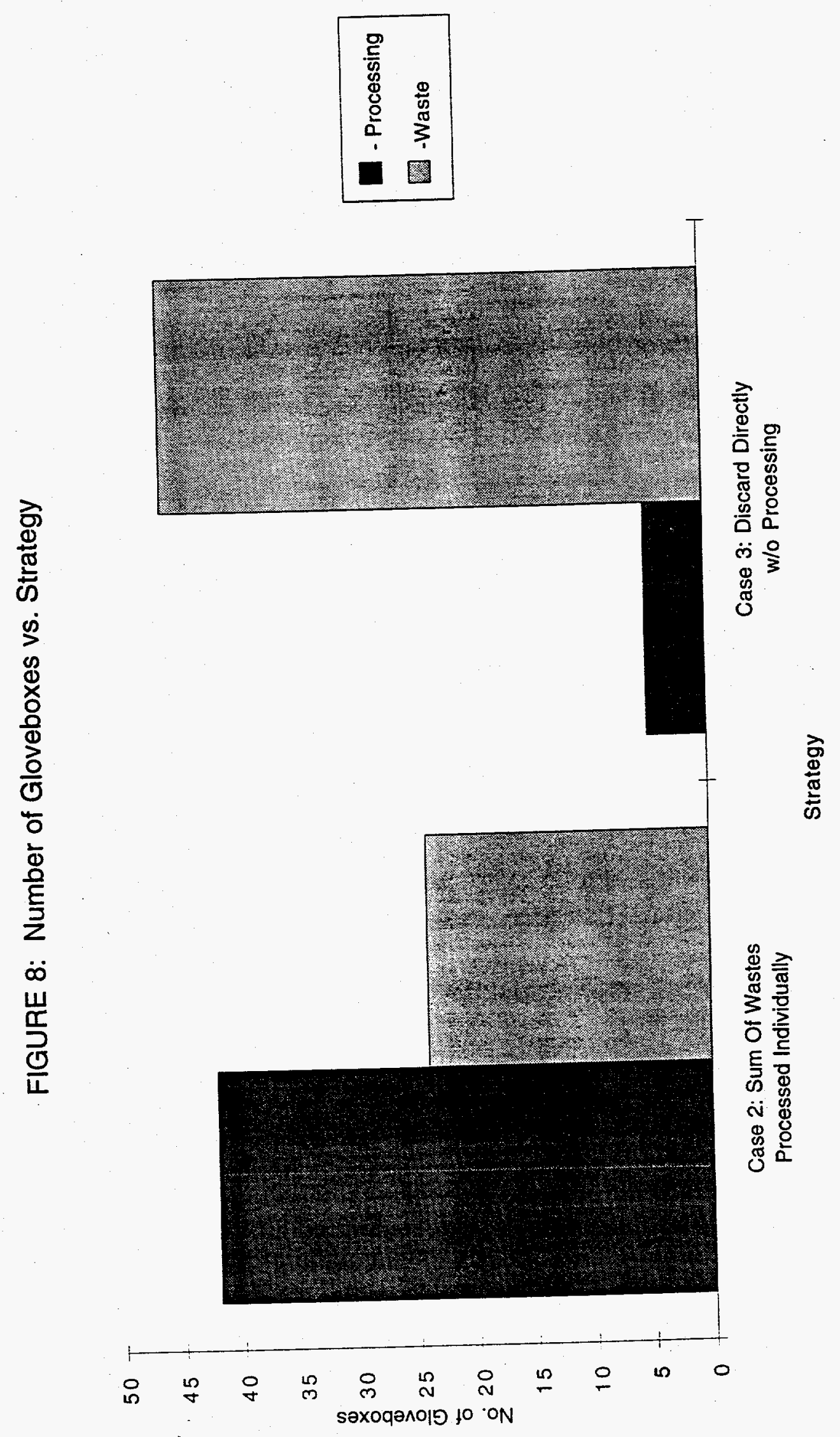


FIGURE 9: Costs - Process Wastes vs. Discard Directly

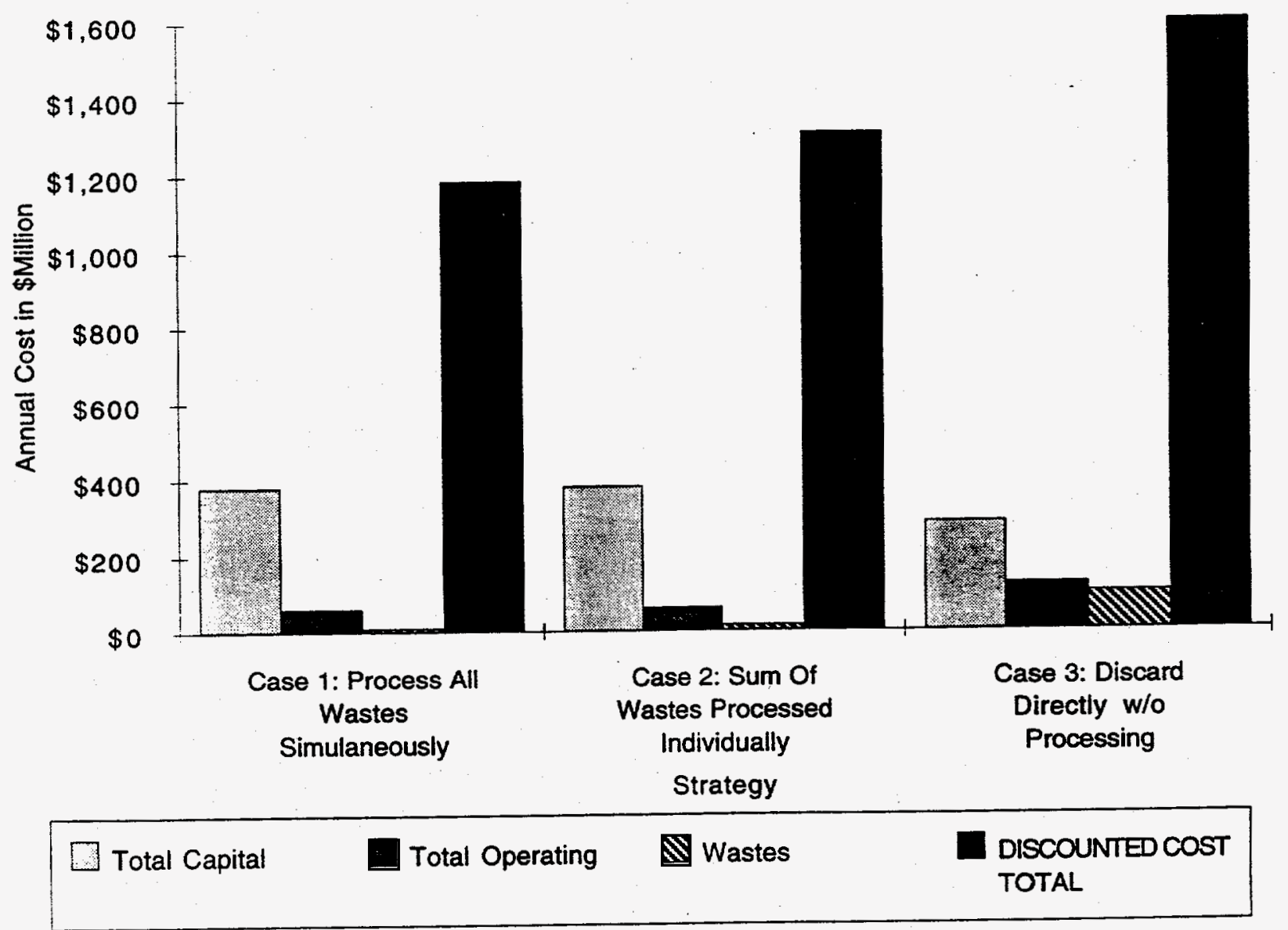


FIGURE 10: Facility Size - Process Wastes vs Discard Directly

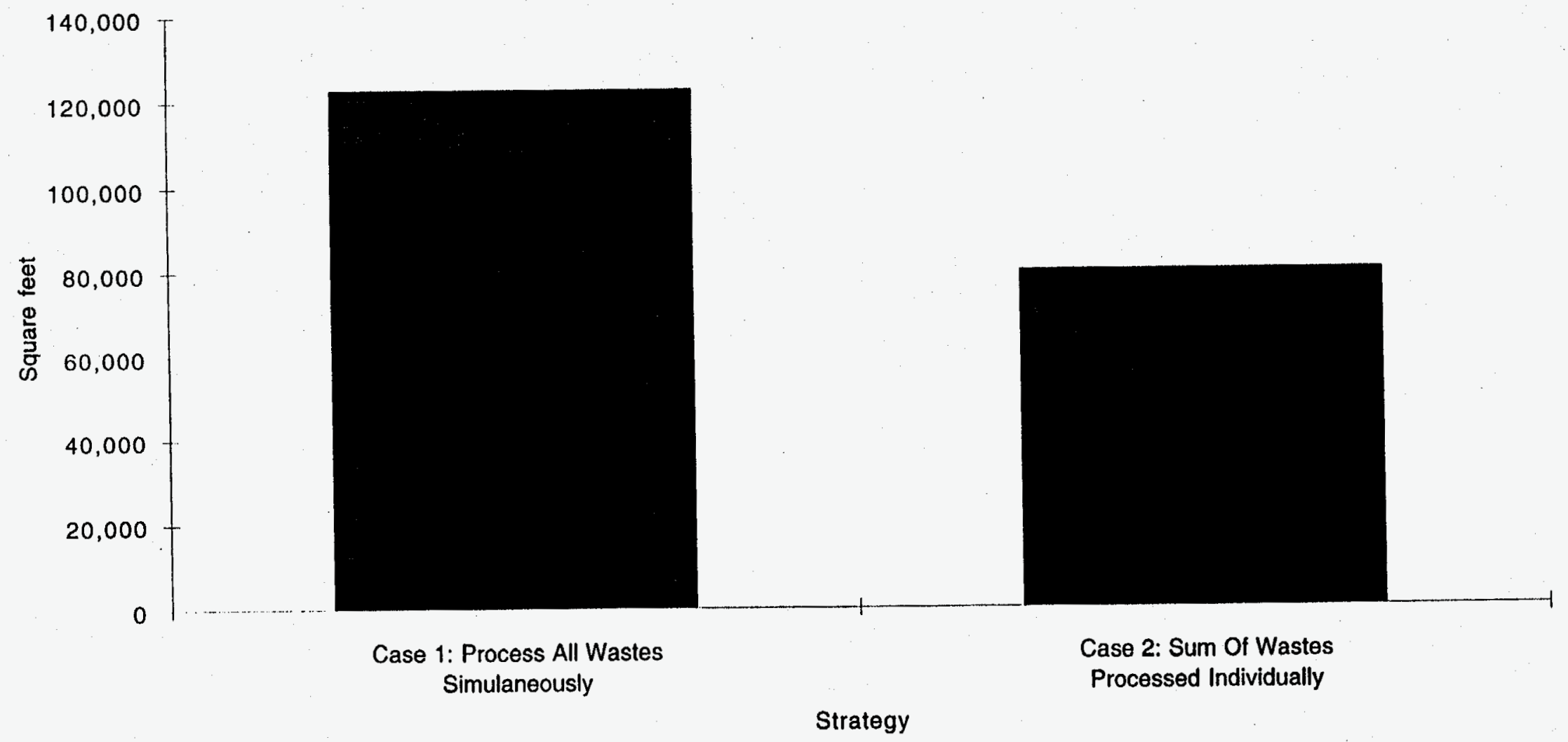


ROCKY FLATS -- Process Residues

\section{Recovery}

Nitrate

PAGE 1 OF 4

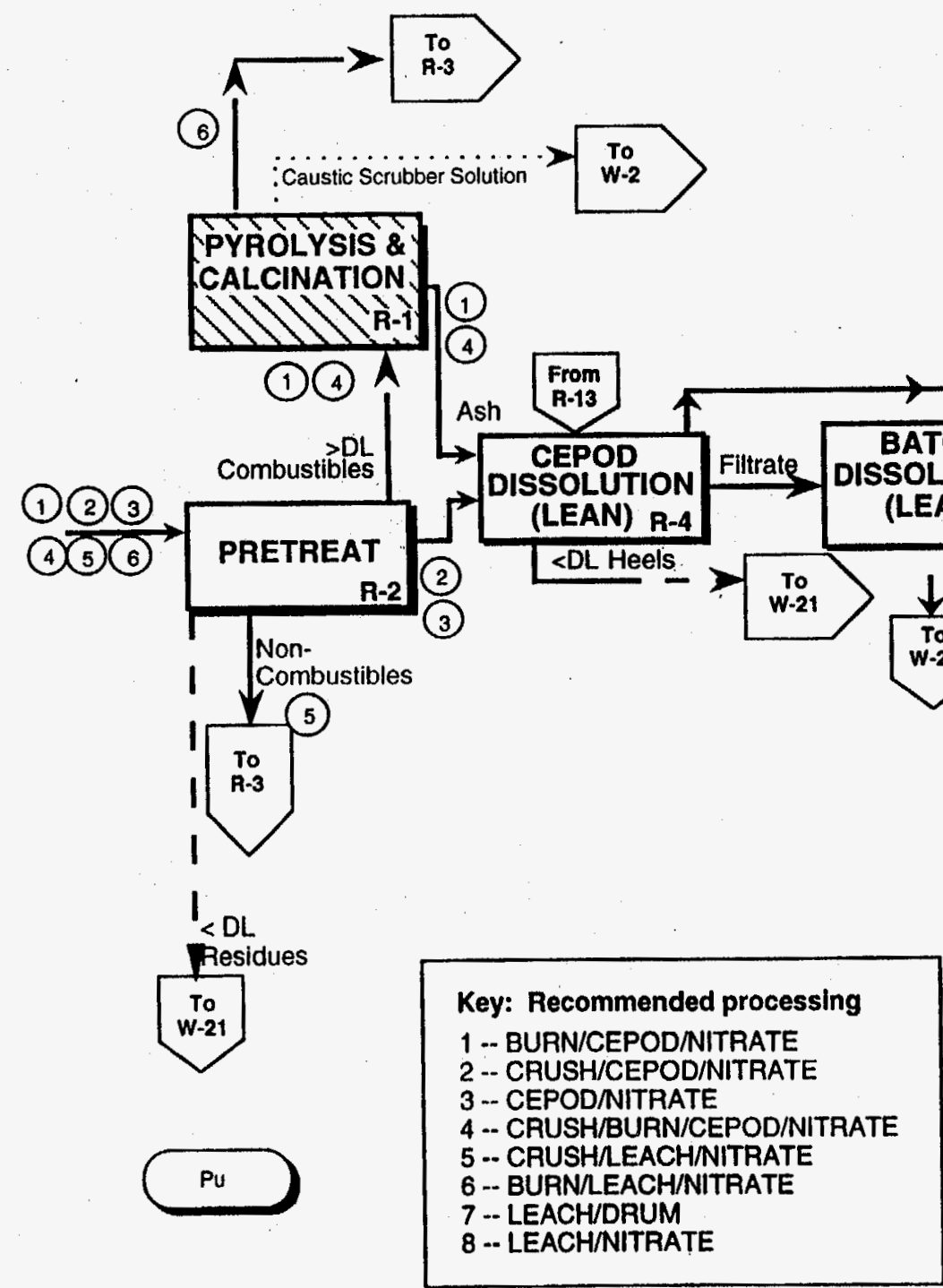

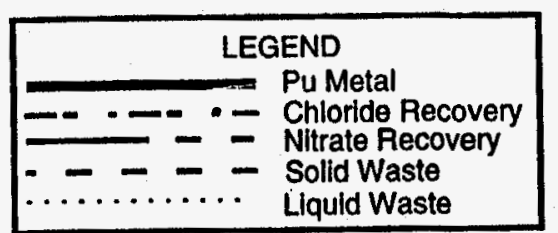

CDL Effluent To $w-2$

(7) $V^{\text {Solids }}$ Filtrate

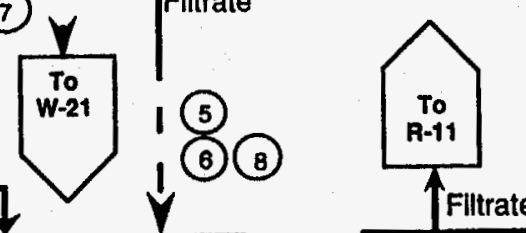

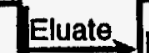
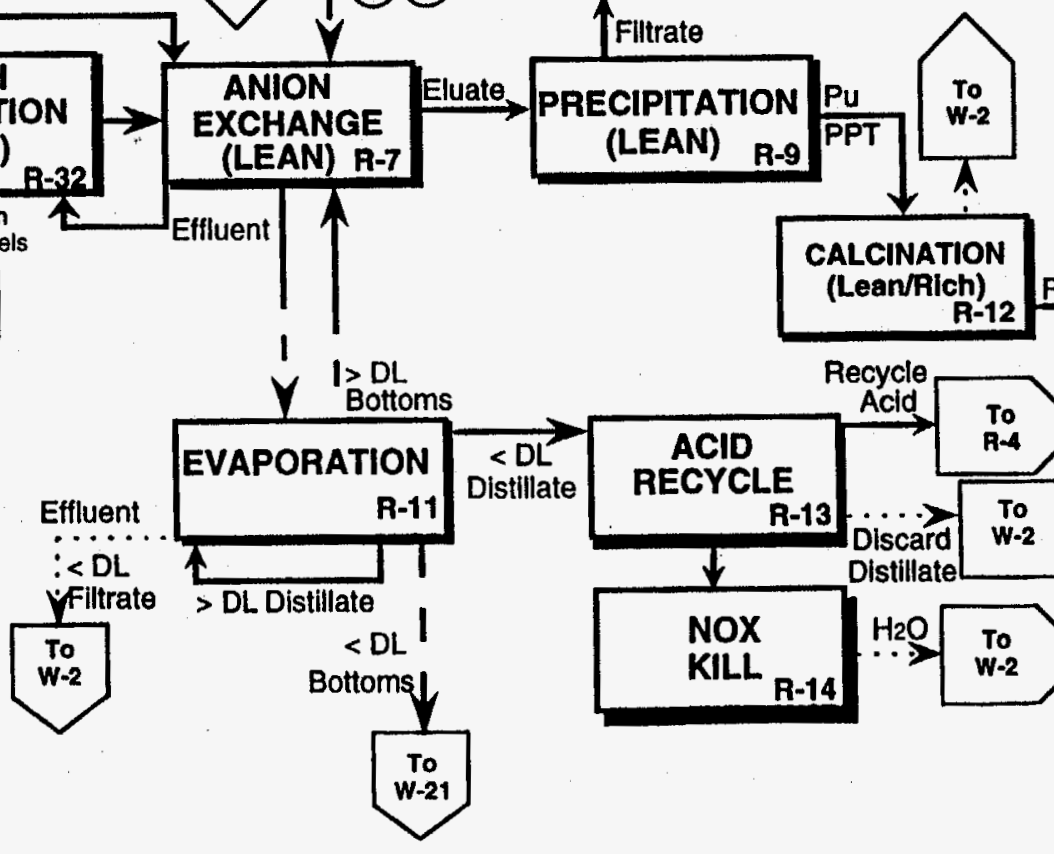

CALCINATION

(Lean/Rich) $\mathrm{PuO}_{2}$ To

Recycle

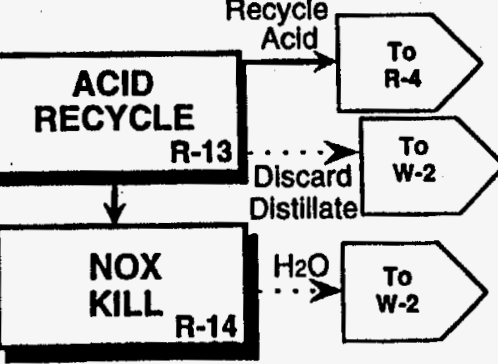
May 1, 1994 Baseline Version
$\mathbf{2 . 2 . 0}$ 


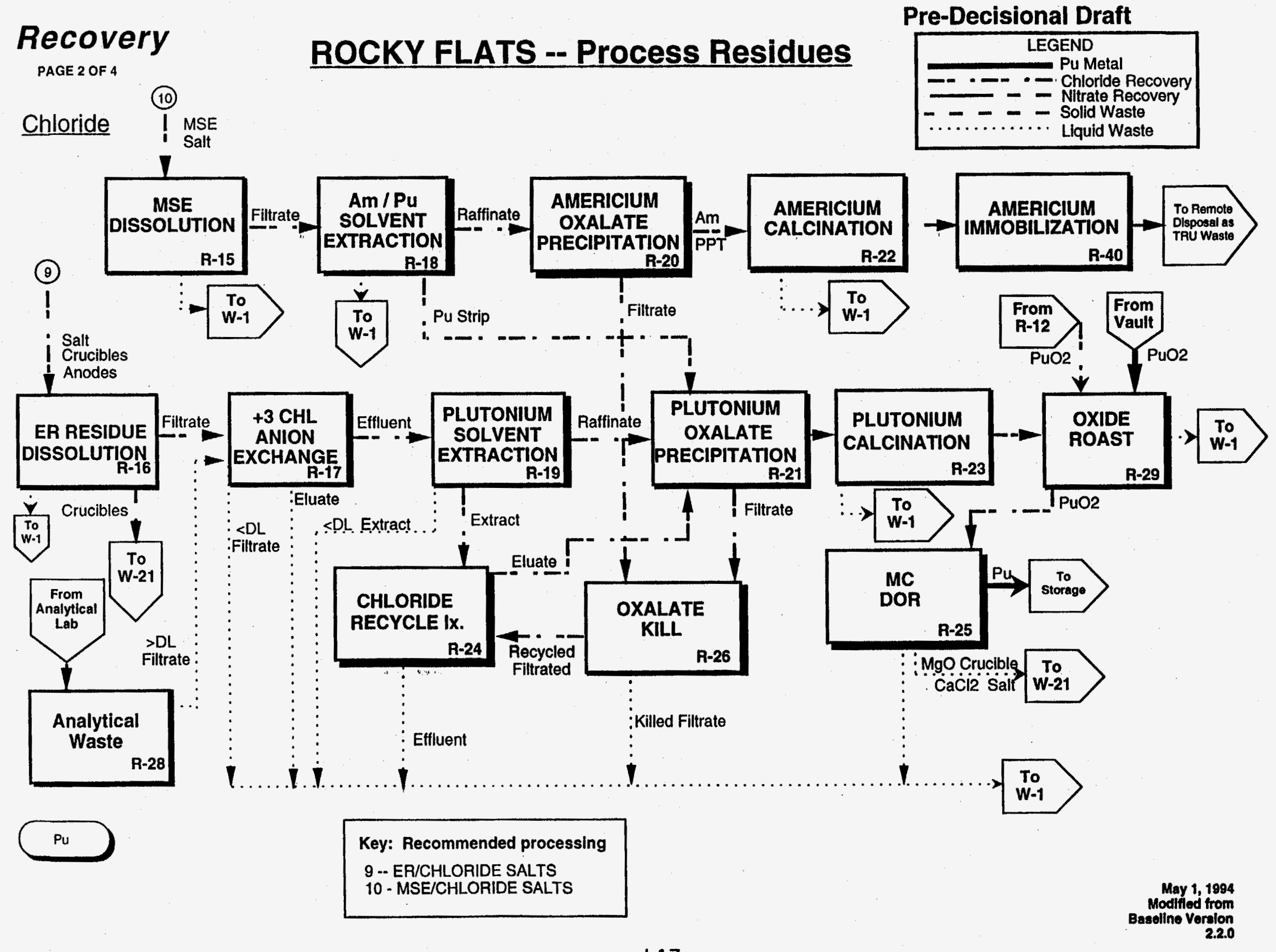




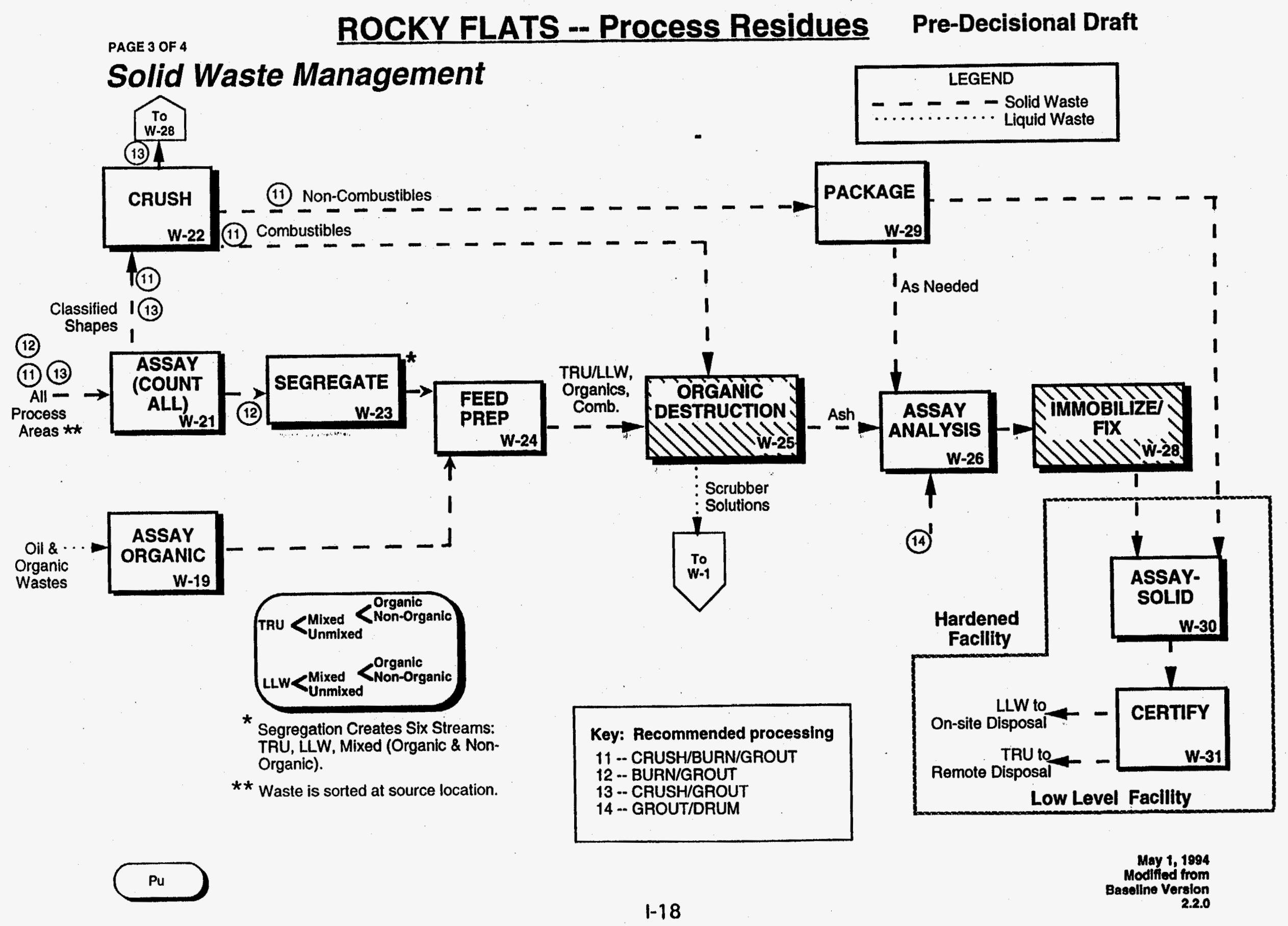


PAGE 4 OF 4

\section{Aqueous Waste Management ROCKY FLATS -- Process Residues}

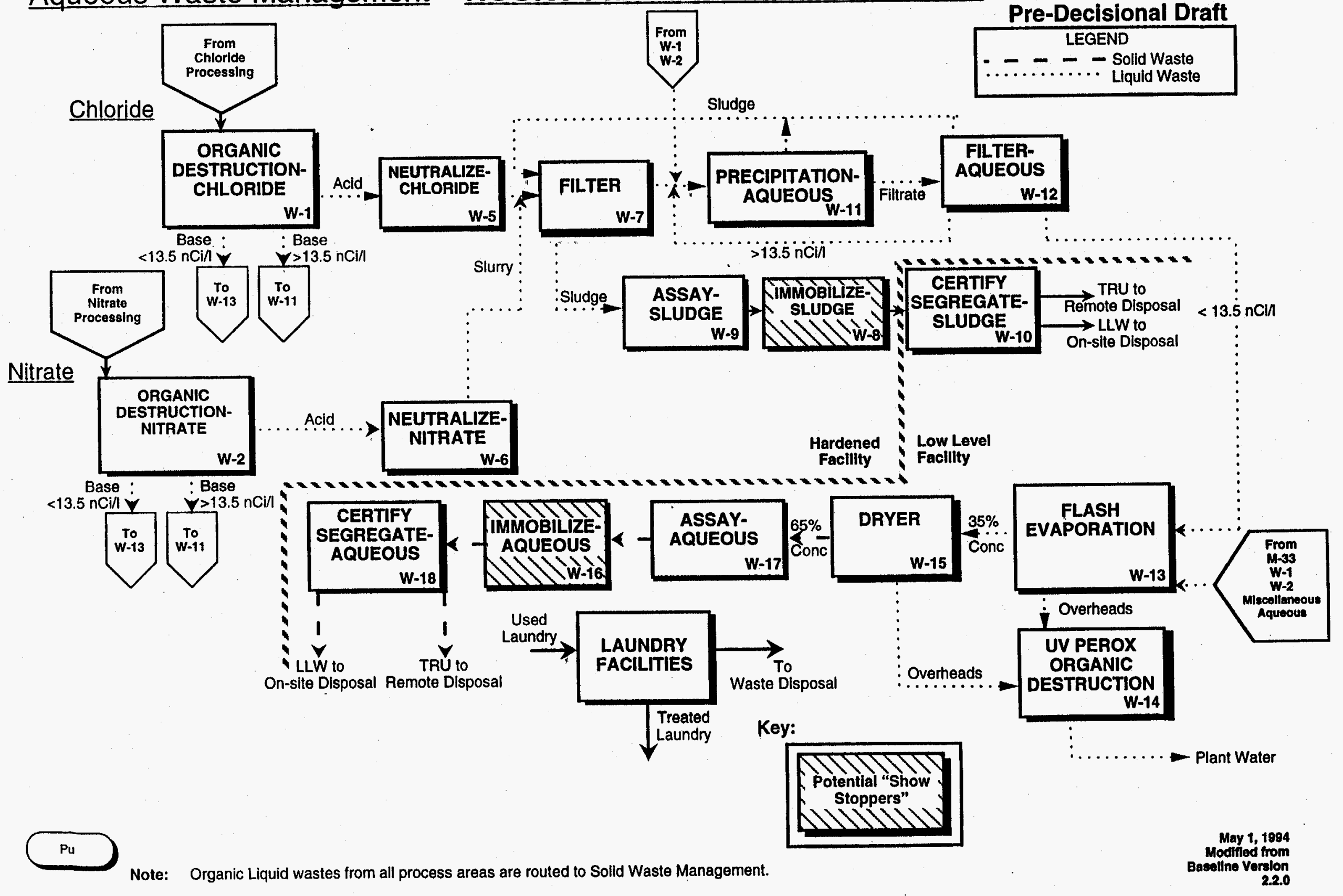




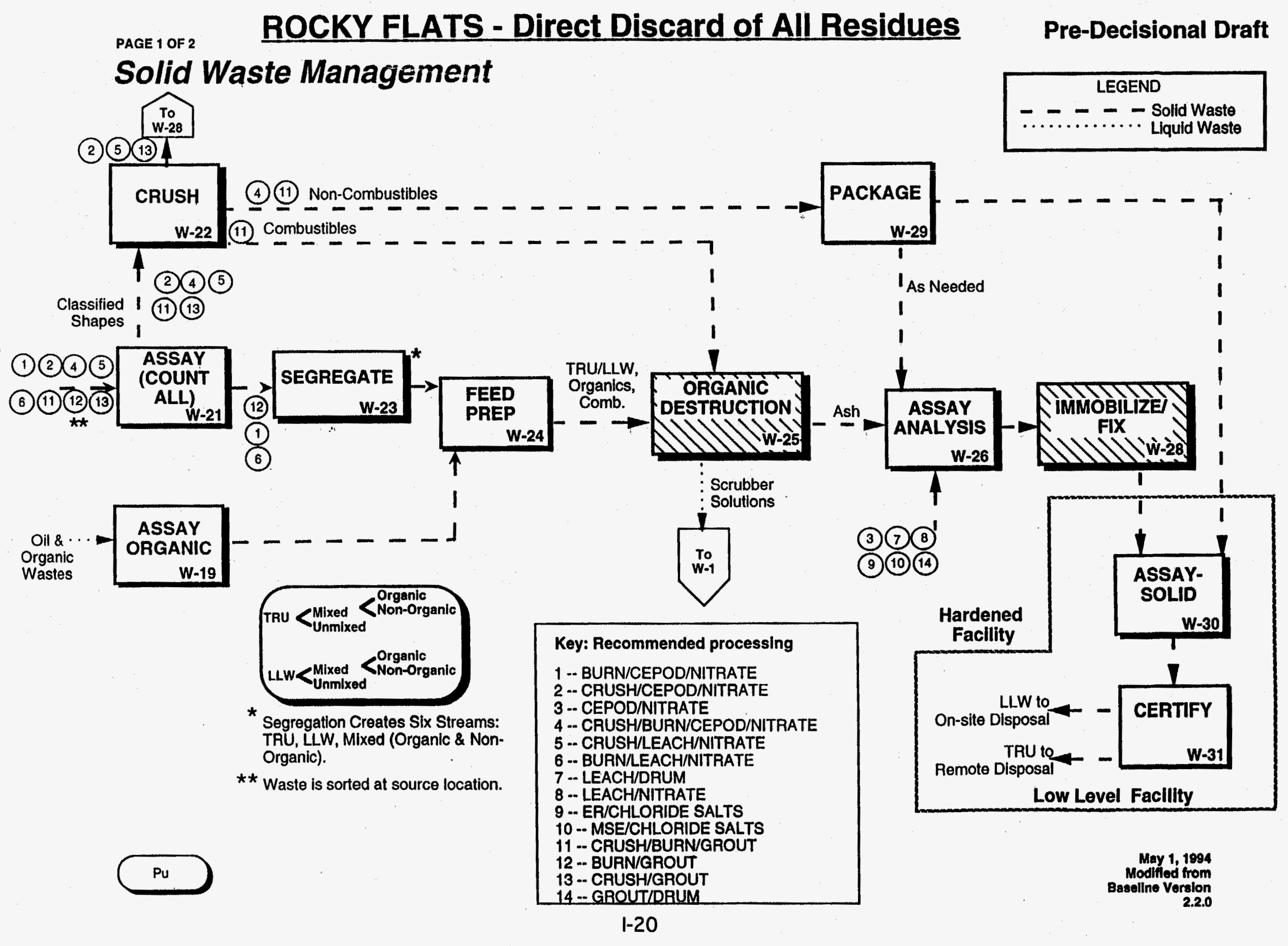




\section{Aqueous Waste Management}

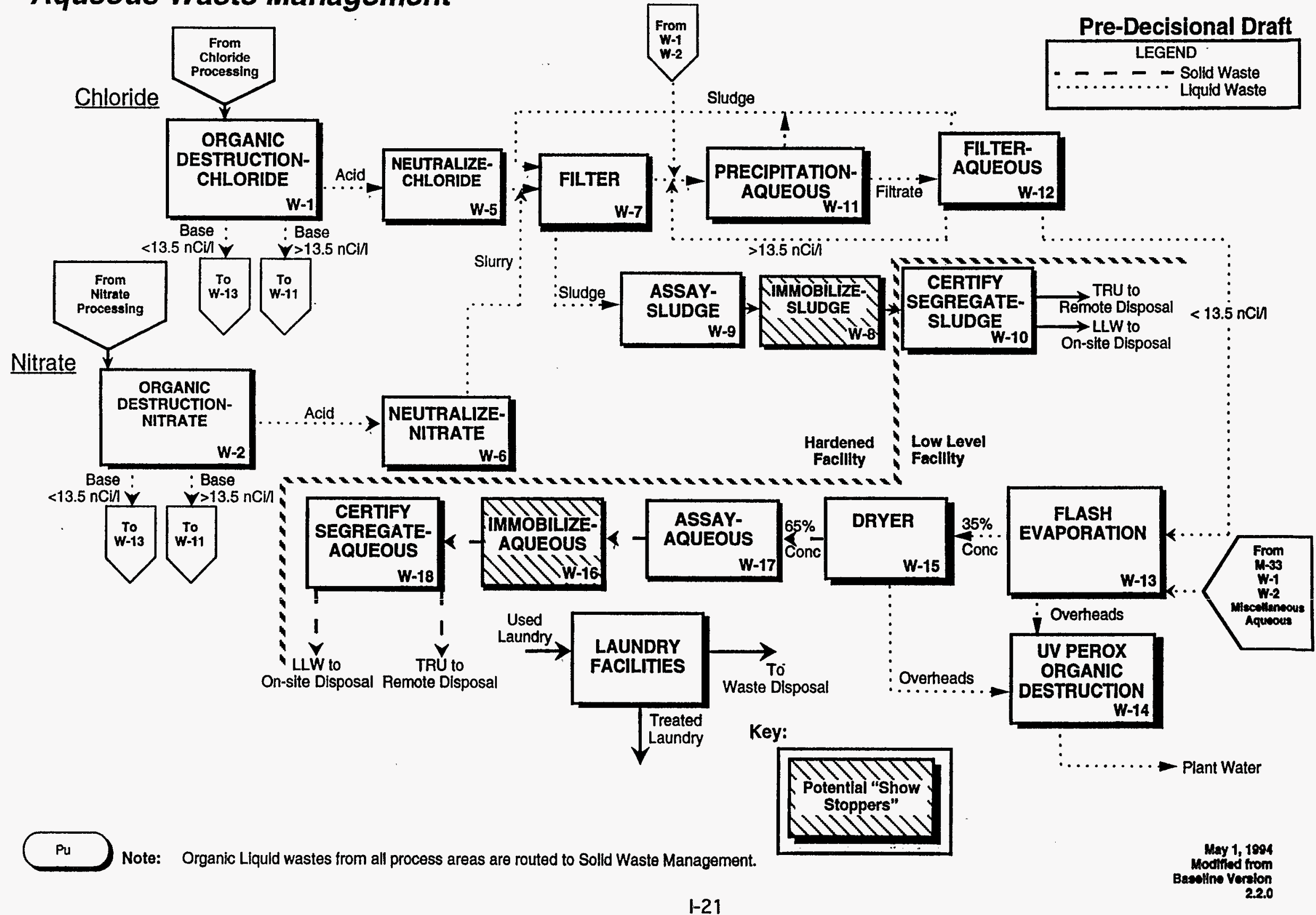

